\title{
Unedited in vivo detection and quantification of $\gamma$-aminobutyric acid in the occipital cortex using short-TE MRS at $3 \mathrm{~T}$
}

\author{
Jamie Near ${ }^{a, b, c *}$, Jesper Anderssonc, Eduard Maron ${ }^{d}$, Ralf Mekle ${ }^{\text {e,f }}$, \\ Rolf Gruetter ${ }^{\text {f,g }}$, Philip Cowen ${ }^{b}$ and Peter Jezzard ${ }^{c}$
}

\begin{abstract}
Short-TE MRS has been proposed recently as a method for the in vivo detection and quantification of $\gamma$-aminobutyric acid (GABA) in the human brain at $3 \mathrm{~T}$. In this study, we investigated the accuracy and reproducibility of short-TE MRS measurements of GABA at $3 \mathrm{~T}$ using both simulations and experiments. LCModel analysis was performed on a large number of simulated spectra with known metabolite input concentrations. Simulated spectra were generated using a range of spectral linewidths and signal-to-noise ratios to investigate the effect of varying experimental conditions, and analyses were performed using two different baseline models to investigate the effect of an inaccurate baseline model on GABA quantification. The results of these analyses indicated that, under experimental conditions corresponding to those typically observed in the occipital cortex, GABA concentration estimates are reproducible (mean reproducibility error, $<20 \%$ ), even when an incorrect baseline model is used. However, simulations indicate that the accuracy of GABA concentration estimates depends strongly on the experimental conditions (linewidth and signal-to-noise ratio). In addition to simulations, in vivo GABA measurements were performed using both spectral editing and short-TE MRS in the occipital cortex of 14 healthy volunteers. Short-TE MRS measurements of GABA exhibited a significant positive correlation with edited GABA measurements $(R=0.58, p<0.05)$, suggesting that short-TE measurements of GABA correspond well with measurements made using spectral editing techniques. Finally, within-session reproducibility was assessed in the same 14 subjects using four consecutive short-TE GABA measurements in the occipital cortex. Across all subjects, the average coefficient of variation of these four GABA measurements was $\mathbf{8 . 7} \pm \mathbf{4 . 9 \%}$. This study demonstrates that, under some experimental conditions, short-TE MRS can be employed for the reproducible detection of GABA at $3 \mathrm{~T}$, but that the technique should be used with caution, as the results are dependent on the experimental conditions. Copyright $\odot 2013$ John Wiley \& Sons, Ltd.
\end{abstract}

Keywords: $\gamma$-aminobutyric acid (GABA); spin-echo full-intensity acquired localised (SPECIAL); MRS; short-TE; spectral editing

\section{INTRODUCTION}

$\gamma$-Aminobutyric acid (GABA) is the primary inhibitory neurotransmitter in the mammalian brain and plays an important role in the regulation of neuronal activity (1). Altered tissue GABA levels have been observed in various pathologies, including both epilepsy (2) and major depression $(3,4)$, and individual variations in tissue GABA levels of healthy individuals have been shown to correlate with both functional MRI activity $(5,6)$ and behaviour

\footnotetext{
* Correspondence to: J. Near, FMRIB Centre, Nuffield Department of Clinical Neurosciences, John Radcliffe Hospital, Headington, Oxford OX3 9DU, UK. E-mail: jnear@fmrib.ox.ac.uk

Faculty of Medicine, Imperial College, London, UK
}

(7,8). In vivo MRS detection of GABA is challenging because of its relatively low concentration and the large overlapping resonances from other metabolites and macromolecules (MMs). As a result, GABA detection is most commonly performed using spectral editing techniques, which enable the selective observation of GABA by separation of the C4-GABA multiplet from the background of overlapping resonances (9-12). Of the available spectral editing methods, probably the most commonly used is the Mescher-Garwood point-resolved spectroscopy (MEGA-PRESS)

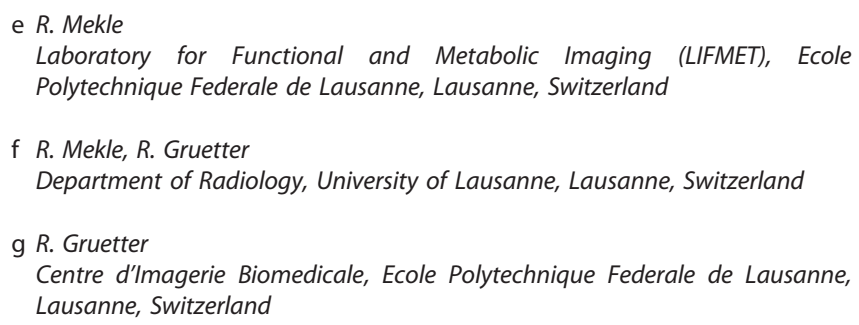


technique (10), which combines MEGA editing (10) with PRESS localisation (13) to achieve reliable quantitative measurements of GABA concentrations within a localised region of tissue. Studies have shown that the MEGA-PRESS technique provides reproducible GABA measurements, with intra-subject reproducibility values in the range $7-12 \%(14,15)$. However, this acquisition method also has a number of associated drawbacks. First, when optimised for the observation of GABA, the MEGA-PRESS technique does not enable the optimal detection of many other metabolites simultaneously. Thus, it provides a very limited amount of useful metabolic information. Second, because the MEGA editing scheme makes use of narrow-band frequency-selective pulses, it is very sensitive to $B_{0}$ field drift, and small drifts of only a few hertz can affect significantly the quantification accuracy $(12,16)$. Third, the technique is inefficient; the observed GABA signal in the MEGA-PRESS experiment typically consists of less than $40 \%$ of the available signal from just one of GABA's three methylene groups (16). The remainder is lost in the editing process.

Short-TE MRS provides a possible alternative approach for the detection of GABA, and may provide several advantages over the more standard spectral editing techniques. Specifically, short-TE MRS enables the detection of a large number of metabolites (including GABA) simultaneously, thus increasing the available amount of metabolic information. Furthermore, in contrast with spectral editing techniques, short-TE MRS is relatively insensitive to $B_{0}$ field drift and is highly efficient as it minimises signal decay from $T_{2}$ relaxation and scalar coupling phase evolution. The detection and quantification of GABA using short-TE MRS in combination with LCModel analysis has been demonstrated previously, but has been mainly restricted to rodent studies and/or very high field strengths ( $\geq 7$ T) (17-20). Recently, however, Mekle et al. (21) demonstrated short-TE (6 ms) MRS detection of GABA at $3 \mathrm{~T}$ in the occipital cortices of six human subjects, with an average Cramer-Rao lower bound (CRLB) uncertainty of $8 \%$. This suggests that reliable GABA detection is feasible at clinical field strengths, without the need for spectral editing. Following this promising initial study, further investigation is required to confirm the initial results and to validate the use of short-TE MRS for the detection of GABA at $3 \mathrm{~T}$.

Therefore, the purpose of this study was to assess the accuracy and reproducibility of GABA detection using the short-TE approach under normal experimental conditions, and to investigate how a change in the experimental conditions, such as linewidth (LW) and signal-to-noise ratio (SNR), influence the accuracy and reproducibility of this technique. These investigations were performed using a methodology similar to that described by Hancu $(22,23)$, namely a $3-T$, short-TE spectrum was simulated by combining metabolite basis spectra in approximate physiological concentrations followed by the addition of noise. The simulated spectrum was then analysed using LCModel, and the resulting metabolite concentration estimates were compared with the known concentrations in the simulated input spectrum. The above procedure was repeated many times to enable the estimation of the overall accuracy and reproducibility of the GABA measurements. In addition to the simulated GABA measurements described above, two in vivo experiments were performed to further evaluate the reproducibility of short-TE MRS detection of GABA. First, in vivo GABA measurements were performed using both spectral editing and short-TE MRS in the occipital cortex of 14 healthy volunteers, and the GABA concentration estimates from short-TE MRS were compared with the gold standard edited GABA measurement. Finally, within-session reproducibility was assessed in the same 14 subjects using four consecutive short-TE GABA measurements in the occipital cortex.

\section{METHODS}

\section{Simulated spectra}

A complete set of metabolite basis spectra consisting of 22 individual metabolites (Table 1) was simulated using an in-house MATLAB-based implementation (MathWorks, Natick, MA, USA) of the density matrix formalism (12). All metabolite chemical shifts and coupling constants were taken from Govindaraju et al. (24), with the exception of GABA, which was defined using the modified spin system parameters provided by Kaiser et al. (25). Metabolites were simulated under the influence of an ideal spin-echo sequence to approximate the short-TE spin-echo fullintensity acquired localised (SPECIAL) technique (21) (2048 points; spectral width, $2000 \mathrm{~Hz}$; TE $=8.5 \mathrm{~ms}$ ) with a field strength of $3 \mathrm{~T}$. Lipid and MM signals were simulated using the same Gaussian basis functions that are simulated by default within the LCModel software (Table 2). A residual water basis spectrum, modelled as a two-proton singlet at $4.7 \mathrm{ppm}$, was also simulated. All basis spectra were line broadened according to the desired LW of the final spectrum, and an additional line-broadening factor was applied to lipid and MM basis spectra, as specified in Table 2 and in the LCModel user manual. Following line broadening, a simulated spectrum was generated by combining all basis spectra in approximate in vivo concentrations. The average metabolite input concentrations were estimated from the literature (24) and are specified in Table 1, whereas the average lipid and MM input concentration values (specified in Table 2) were estimated from 12 LCModel outputs of previously obtained in vivo datasets

Table 1. List of simulated metabolites and average concentrations

\begin{tabular}{lc|} 
Metabolite & Average concentration (mM) \\
\hline Alanine & 0.5 \\
Ascorbate & 0.8 \\
Aspartate & 1.5 \\
Creatine & 5.25 \\
Phosphocreatine & 4.75 \\
$\gamma$-Aminobutyric acid & 1.2 \\
Glutamine & 3.2 \\
Glutamate & 9.5 \\
Glutathione & 1.5 \\
Myo-inositol & 6 \\
Lactate & 0.4 \\
N-Acetylaspartate & 12 \\
Scyllo-inositol & 0.45 \\
Taurine & 1.5 \\
Glucose & 1.0 \\
$N$-Acetylaspartylglutamate & 1.5 \\
Phosphoethanolamine & 1.3 \\
Glycerophosphocholine & 1.0 \\
Phosphocholine & 0.6 \\
Glycine & 0.7 \\
Serine & 0.4 \\
$\beta$-Hydroxybutyrate & 0.1
\end{tabular}


Table 2. Details of simulated lipid (Lip) and macromolecule (MM) basis spectra

\begin{tabular}{|lcccc|}
\hline Lipid/macromolecule & Frequency $(\mathrm{ppm})$ & Line-broadening factor $(\mathrm{Hz})$ & Amplitude (\# protons) & Concentration (mM) \\
\hline MM09 & 0.91 & 21.0 & 3 & 1.33 \\
Lip20 & 2.04 & 24.6 & 0.67 & 1.0 \\
& 2.25 & 18.5 & 0.87 & 1.33 \\
MM20 & 1.95 & 24.6 & 0.33 & \\
& 2.08 & 22.2 & 0.33 & \\
& 2.25 & 24.6 & 0.40 & \\
MM12 & 1.95 & 18.5 & 2.0 & 3.0 \\
MM14 & 3.00 & 24.6 & 2.0 & 8.0 \\
MM17 & 1.21 & 24.6 & 2.0 & 4.0 \\
\hline
\end{tabular}

acquired in the occipital cortex of normal subjects using the relevant pulse sequence and timing parameters. As with the metabolites, the concentrations of each of the six MMs and lipid signals were allowed to vary independently of each other. Normally distributed random noise was then added to the simulated spectrum to achieve the desired SNR, which was defined as the maximum metabolite peak height divided by the standard deviation of the added noise. For a given SNR and LW, the above procedure was repeated 500 times with different noise seeds. In each of the 500 repetitions, to account for normal subject-to-subject variation, the input concentration of each basis spectrum was allowed to vary randomly with a standard deviation of $15 \%$ of its mean value. The only exception to this was the residual water signal, which was assigned an average value of $0 \mathrm{~mm}$ and a standard deviation of $20 \mathrm{~mm}$.

Up to this point in the simulation process, 500 simulated spectra will have been generated in which the experimental conditions (LW and SNR) are identical, and the relative metabolite concentrations will vary as they might in a normal human population. Then, the same procedure was repeated for different sets of experimental conditions. In total, every combination of 11 different LW values (ranging from 2 to $12 \mathrm{~Hz}$ in integer steps) and 18 different SNR values (ranging from 50 to 900 , in steps of 50) was tested, for a total of 198 experimental conditions and 99000 simulated spectra. Each spectrum was then processed twice in LCModel; once without a baseline fitting component (achieved by setting 'NOBASE $=\mathrm{T}^{\prime}$ ) and once using the default LCModel baseline setting, in which LCModel attempts to find the smoothest possible baseline that is still consistent with the data. For analysis of the simulated data in this study, the baseline-free analysis is the more appropriate option, as all of the peaks in the simulated spectra (with the exception of the tail of the residual water peak) should be accounted for by the basis set. However, a second analysis was performed using the default baseline setting to examine the effect of an incorrect baseline model on the accuracy and reproducibility of GABA concentration estimates. Both LCModel analyses were performed using the same basis set as was employed to generate the simulated datasets and the LCModel estimates of the metabolite concentrations, and CRLB uncertainties were recorded for each analysis. Critically, the metabolite input concentrations are precisely known for each simulated spectrum; therefore, it is possible to assess the accuracy and reproducibility of the metabolite concentration estimates obtained from LCModel.

\section{Assessment of measurement bias and reproducibility}

For each set of experimental conditions, the measurement bias and reproducibility were assessed. Measurement bias was assessed by calculating the mean estimation error $\left(\% E_{\mathrm{E}}\right)$, which was defined as the average percentage difference between the estimated and actual GABA concentration values:

$$
\% E_{E}=\frac{1}{N} \sum_{i}^{N} \frac{C_{\text {estimated }_{i}}-C_{\text {actual }_{i}}}{C_{\text {actual }_{i}}} \times 100=\frac{1}{N} \sum_{i}^{N} \frac{E_{i}}{C_{\text {actual }_{i}}} \times 100
$$

where $C_{\text {estimated }}$ is the concentration estimate from LCModel, $C_{\text {actual }}$ is the known input concentration, updated to correctly account for subject-to-subject variation in the data, $E$ is the difference between the estimated and actual metabolite concentrations and $N$ is the total number of simulated spectra per experimental condition (500). Reproducibility was assessed by calculating the reproducibility error $\left(\% E_{R}\right)$. This was performed by first plotting the measured GABA concentrations versus the actual GABA input concentrations and performing a linear least-squares fit to the data. $\% E_{\mathrm{R}}$ was then given by the standard deviation of the values of the fitted residuals, divided by the average of the estimated concentration values:

$$
\% E_{R}=\frac{\sqrt{\frac{1}{N} \sum_{i}^{N}\left(C_{\text {estimated }_{i}}-C_{\text {fit }_{i}}\right)^{2}}}{\frac{1}{N} \sum_{i}^{N} C_{\text {estimated }_{i}}} \times 100=\frac{\sqrt{\frac{1}{N} \sum_{i}^{N} e_{i}^{2}}}{\frac{1}{N} \sum_{i}^{N} C_{\text {estimated }_{i}}} \times 100
$$

where $C_{\text {fit }}$ is the concentration calculated from the linear data fit described above and $e_{i}$ are the fitted residuals. By this definition, $\% E_{\mathrm{R}}$ is expressed as a percentage and provides an approximate measure of the coefficient of variation. It should be noted that, because the reproducibility error $\% E_{\mathrm{R}}$ is expressed relative to the average measured value $C_{\text {estimated, }}$ its value is dependent on the measurement bias. Therefore, it is informative to define a second measure of the reproducibility error $\left(\% E_{\mathrm{R} 2}\right)$, which is independent of measurement bias. This is achieved by, instead, expressing the reproducibility error relative to the average actual concentration value $C_{\text {actual }}$. Although this is no longer a true measurement of the coefficient of variation, it provides a means of assessing the reproducibility independently of any potential measurement bias. 


\section{In vivo experiments}

All volunteers ( $n=14$; age, $23.3 \pm 5.4$ years; eight women, six men) provided informed, written consent and were scanned on a 3-T Siemens TIM Trio scanner (Erlangen, Germany) with a body coil transmitter and a 32-channel receive head array. Both shortTE and edited GABA spectra were acquired in the same scan session from the same localised region measuring $2.5 \times 2.5 \times 2.5$ $\mathrm{cm}^{3}$ in the occipital cortex. Shimming was performed using the vendor-provided automated shim tool. Short-TE MR spectra were acquired using the SPECIAL sequence (4096 points; spectral width, $4000 \mathrm{~Hz} ; \mathrm{TR} / \mathrm{TE}=3000 / 8.5 \mathrm{~ms} ; 192$ averages) and edited GABA spectra were acquired using the MEGA-SPECIAL sequence (2048 points; spectral width, $2400 \mathrm{~Hz} ; \mathrm{TR} / \mathrm{TE}=3000 / 68 \mathrm{~ms} ; 192$ averages). The MEGA-SPECIAL sequence was implemented with 20-ms editing pulses and an MM-unsuppressed editing scheme (12). To limit the amount of frequency drift in any single scan, the MEGA-SPECIAL acquisition was broken into three blocks of 64 averages, and a system frequency adjustment was performed prior to the start of each acquisition block. For both short-TE and edited acquisitions, outer volume suppression was applied prior to each scan to saturate spins on all six sides of the region of interest, and VAPOR (variable power radiofrequency pulses with optimised relaxation delays) water suppression was used (19). Finally, eight averages of waterunsuppressed data were also acquired with the same outer volume suppression scheme as above.

\section{Post-processing and analysis}

The same post-processing chain was applied to both the edited and short-TE datasets. First, 32-channel data were recombined in a weighted fashion, with coil weights and phases determined using the magnitude and phase, respectively, of the first time domain point of the water-unsuppressed data. Following coil recombination, the subspectra resulting from SPECIAL preinversion on/off scans were subtracted from each other, resulting in properly localised scans. Following subtraction of the subspectra, a strict procedure to remove motion-corrupted scans was employed. To identify motion-corrupted scans, a metric was developed to measure the 'unlikeness' of each scan to the average of all scans. Specifically, an 'unlikeness metric' was calculated for each individual scan by subtracting the scan from the average of all scans (to obtain a difference spectrum) and then taking the root-mean-square of all of the spectral points in the difference spectrum. Scans whose 'unlikeness metrics' fell more than 2.6 standard deviations above the average were deemed to have been corrupted by motion, and were removed. The 2.6 standard deviation threshold was determined from experience to be fairly successful at removing outlier scans without removing uncorrupted averages. Following the removal of motion-corrupted scans, but prior to signal averaging, a frequency and phase drift correction was performed. This was achieved by least-squares fitting of each scan to the first scan in the series, using frequency and phase as adjustment parameters. To reduce computational load, this procedure was performed in the time domain, using only the first $40 \mathrm{~ms}$ of data. For the MEGA-SPECIAL edited data, both the removal of motioncorrupted scans and the frequency and phase alignment were performed separately for edit-on and edit-off spectra, with the constraint that the number of edit-on and edit-off scans removed must be equal. Following frequency and phase alignment of the scans, signal averaging was performed, resulting in a fully processed short-TE spectrum, and fully processed edit-on and edit-off MEGA-SPECIAL data. The edit-on and edit-off scans in the MEGA-SPECIAL data were then manually frequency and phase aligned to minimise the residual choline difference signal, and the edit-on and edit-off scans were then subtracted, resulting in three fully processed difference-edited spectra (one for each of the three acquisition blocks). Finally, the three fully processed MEGA-SPECIAL data blocks were combined using the same automated time domain frequency and phase alignment algorithm as used for drift correction, resulting in a single fully processed difference edited spectrum.

All experimentally acquired short-TE SPECIAL MRS data were analysed in LCModel using the default baseline setting and the same basis set as used for the analysis of the simulated data as described above. Edited MEGA-SPECIAL MRS data were analysed by peak fitting using jMRUI software (26), according to the method described previously (12). Metabolite concentration estimates were corrected for $T_{2}$ relaxation during TE by assuming $T_{2}$ values of 88 and $116 \mathrm{~ms}$ for GABA and creatine, respectively.

Within-session reproducibility was assessed by splitting the previously acquired short-TE SPECIAL data into four equal and consecutive blocks, each containing 48 averages. Each of these four blocks was then pre-processed identically, as described above, with the sole restriction that the same number of averages was discarded from each of the four blocks for removal of motion corruption. Each of the four processed datasets was then analysed using LCModel employing the default baseline setting, and the GABA concentration estimates were recorded. The coefficient of variation of the GABA estimates across the four scan blocks was then calculated for each subject.

For the measurement of SNR in experimental data, the 'signal' was defined as the maximum intensity of the real part of the metabolite signal between 0.2 and $4.2 \mathrm{ppm}$, which always corresponded with the $N$-acetylaspartate (NAA) peak. The 'noise' was calculated by performing a second-order polynomial detrend of each of the spectral regions between $[-2.5,-1.5],[-1.5$, $-0.5],[10,11]$ and $[11,12] \mathrm{ppm}$, and then taking the average of the standard deviations of the real parts of the signals in these regions.

\section{RESULTS}

The contour plots in Figure 1 illustrate the measurement bias and reproducibility errors of GABA estimates as a function of LW and SNR for the simulated data when the baseline component is omitted from LCModel fitting. The measurement bias $\left(\% E_{\mathrm{E}}\right)$, reproducibility error $\left(\% E_{\mathrm{R}}\right)$, average $C R L B$ and absolute reproducibility error $\left(\% E_{R 2}\right)$ are shown in Figures $1 \mathrm{a}-\mathrm{d}$, respectively.

Figure 2 illustrates the same parameters as in Figure 1 when using the default baseline setting in LCModel instead of the baseline-free option. The measurement bias $\left(\% E_{\mathrm{E}}\right)$, reproducibility error $\left(\% E_{R}\right)$, average CRLB and absolute reproducibility error $\left(\% E_{\mathrm{R} 2}\right)$ are shown in Figures $2 a-d$, respectively.

Figure 3a shows a representative in vivo spectrum acquired using the short-TE SPECIAL sequence, together with an anatomical image showing the location of the volume of interest in the occipital cortex. Across the 14 subjects scanned, the average LW was $5.5 \pm 0.8 \mathrm{~Hz}$ and the average SNR was $665 \pm 93$. The average CRLB for GABA, as measured 

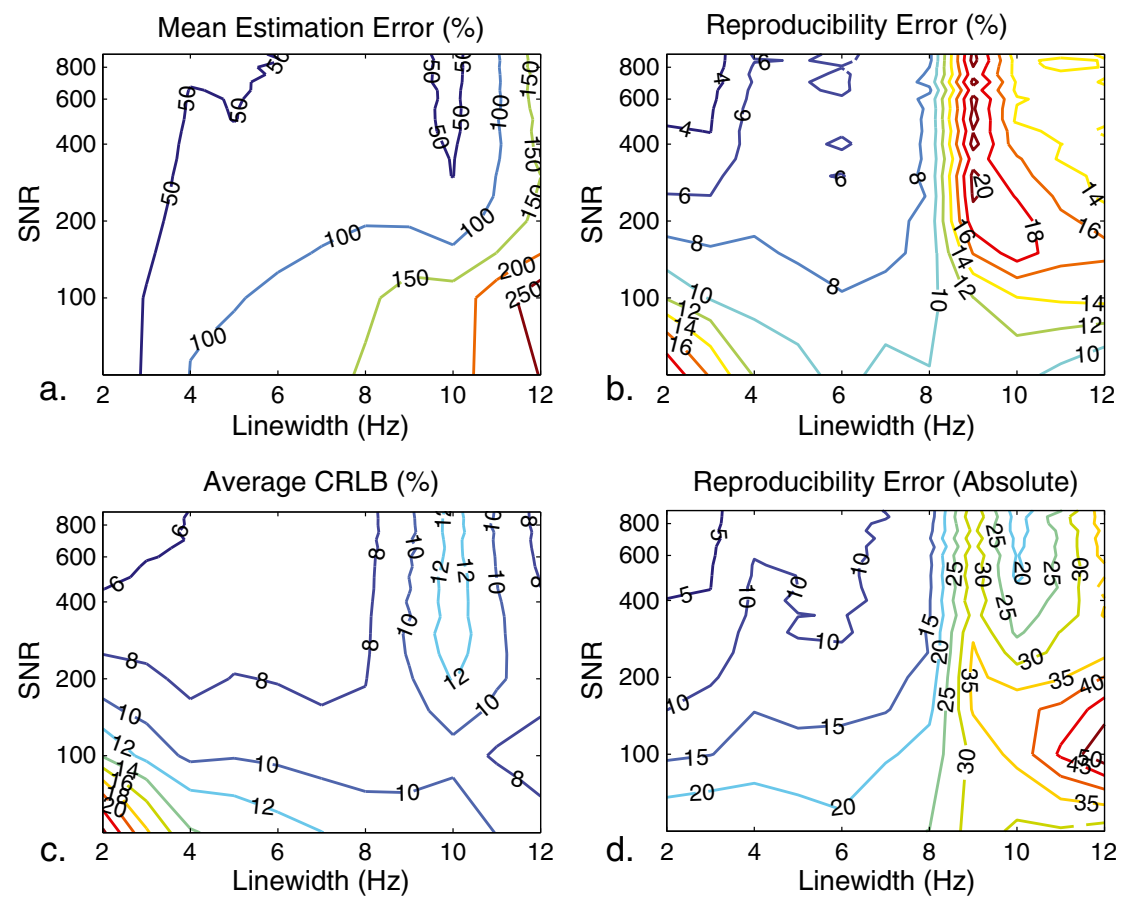

Figure 1. Contour plots showing mean estimation error $\left(\% E_{E}\right)(a)$, reproducibility error $\left(\% E_{R}\right)(b)$, average Cramer-Rao lower bound (CRLB) (c) and absolute reproducibility error $\left(\% E_{\mathrm{R} 2}\right)$ (d) of $\gamma$-aminobutyric acid $(\mathrm{GABA})$ for simulated data as a function of linewidth (LW) and signal-to-noise ratio (SNR). LCModel analysis was performed with no baseline component by setting 'NOBASE $=\mathrm{T}^{\text {'. }}$
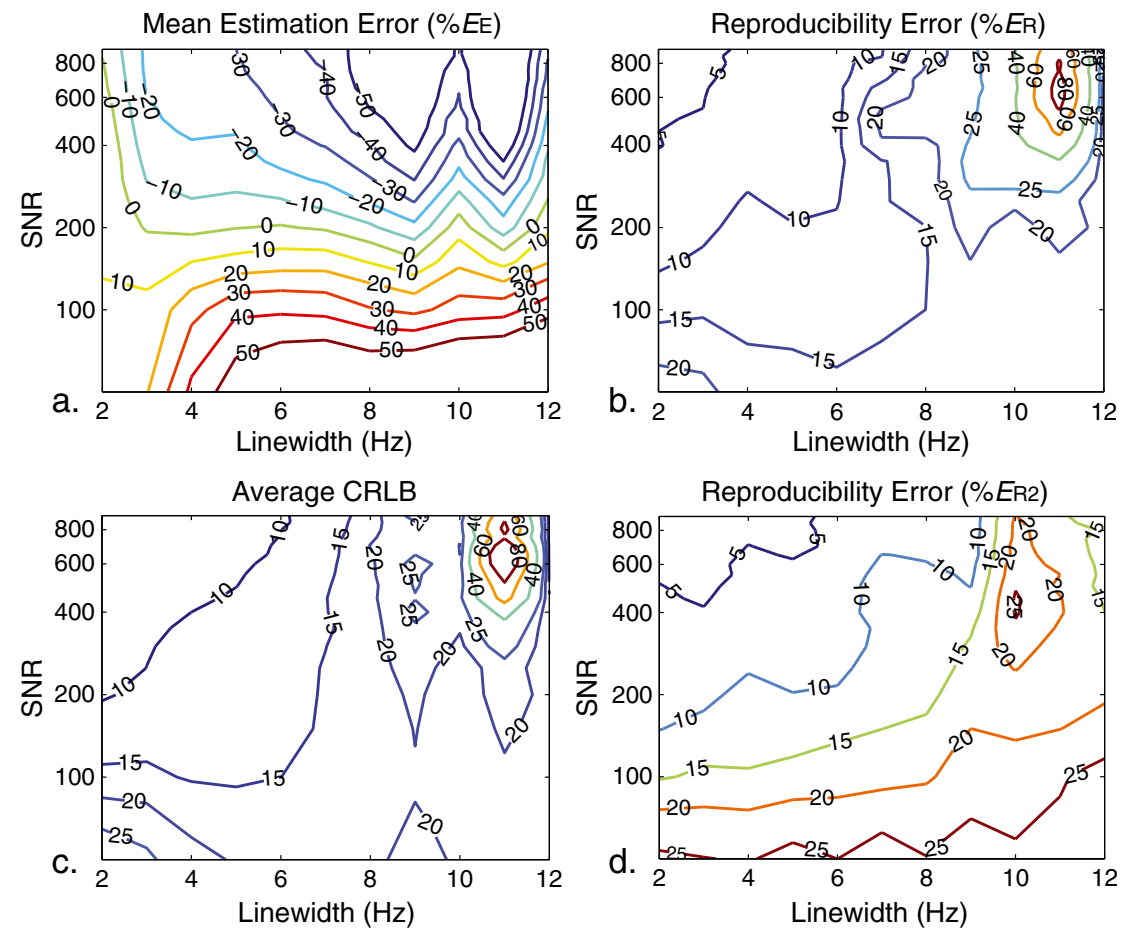

Figure 2. Contour plots showing mean estimation error $\left(\% E_{E}\right)(a)$, reproducibility error $\left(\% E_{R}\right)(b)$, average Cramer-Rao lower bound $(C R L B)(c)$ and absolute reproducibility error $\left(\% E_{\mathrm{R} 2}\right)(\mathrm{d})$ of $\gamma$-aminobutyric acid (GABA) for simulated data as a function of the linewidth (LW) and signal-to-noise ratio (SNR). LCModel analysis was performed using the default baseline model.

using LCModel, was $11.7 \pm 2.8 \%$. Figure $3 \mathrm{~b}$ shows an example of an edited spectrum acquired using the MEGA-SPECIAL sequence in the same subject and voxel.
In Figure 4, the GABA concentrations measured with short-TE SPECIAL MRS are plotted against the edited GABA measurements made using MEGA-SPECIAL. A significant positive linear 


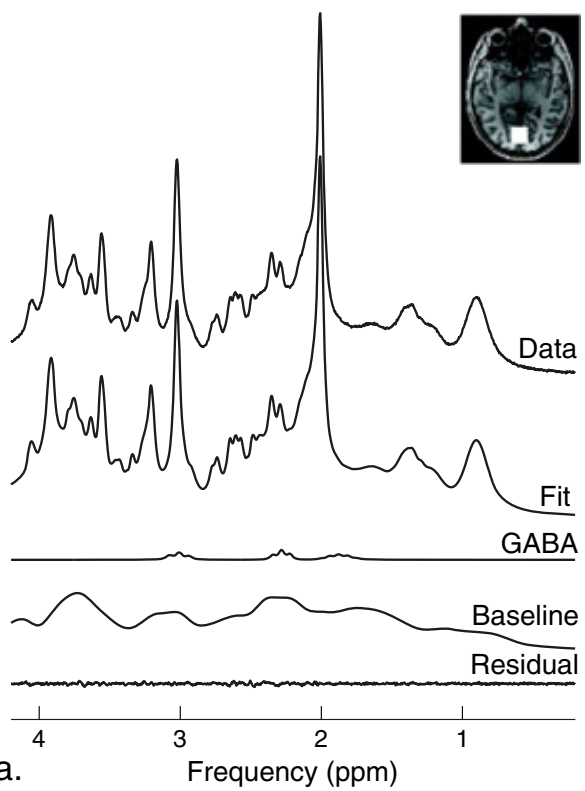

a.

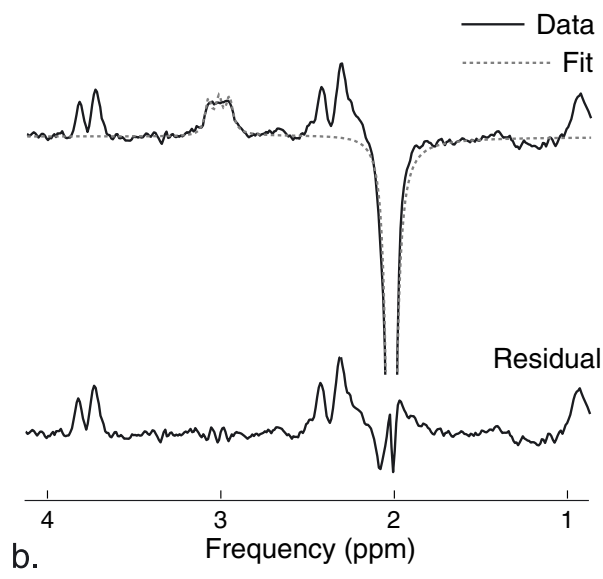

Figure 3. Representative short-TE spin-echo full-intensity acquired localised (SPECIAL) [(a), TR/TE $=3000 / 8.5 \mathrm{~ms}, 192$ averages $]$ and edited Mescher-Garwood (MEGA)-SPECIAL [(b), TR/TE $=3000 / 68$ ms, 192 averages] MRS data from a $2.5 \times 2.5 \times 2.5-\mathrm{cm}^{3}$ volume of interest in the occipital cortex. Both datasets demonstrate very good spectral quality and small residual signals, indicating a good fit.

relationship between the two measures was observed $(R=0.58$, $p<0.05)$.

Figure 5 a shows one of 500 simulated spectra generated with similar experimental conditions to the in vivo data in Figure 4 $(\mathrm{LW}=6 \mathrm{~Hz} ; \mathrm{SNR}=650)$. Figure $5 \mathrm{~b}$ shows the estimated $\mathrm{GABA}$ concentrations from each of the 500 spectra (measured using the default baseline setting in LCModel) plotted versus the actual GABA concentrations. Under these experimental conditions, GABA concentrations are systematically underestimated, and a strong positive correlation is observed between the estimated and actual GABA concentrations $(R=0.84)$. For these simulated data, the calculated values of $\% E_{\mathrm{E}}, \% E_{\mathrm{R}}$, average $C R L B$ and $\% E_{\mathrm{R} 2}$ were $-33.7 \%, 8.9 \%, 10.3 \%$ and $5.9 \%$, respectively, when using the default baseline setting of LCModel.

Across the 56 shortened acquisitions generated for the investigation of within-session reproducibility, the average LW was $5.5 \pm 0.8 \mathrm{~Hz}$ and the average SNR was approximately half of the average SNR values for the full acquisitions ( $340 \pm 53$ ).

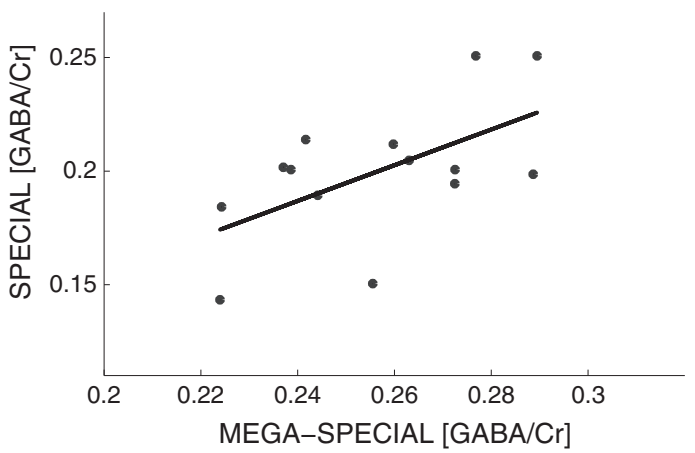

Figure 4. Comparison of $\gamma$-aminobutyric acid/creatine (GABA/Cr) concentration estimates obtained using both short-TE spin-echo full-intensity acquired localised (SPECIAL) (y axis) and edited Mescher-Garwood (MEGA)-SPECIAL ( $x$ axis). A significant positive relationship is observed $(R=0.58, p<0.05)$, suggesting that short-TE SPECIAL measurements correlate well with gold-standard edited measurements of GABA.

The average CRLB for GABA, as measured using LCModel, was $12.6 \pm 2.0 \%$. For simulated data under similar experimental conditions ( $L W=6 ; S N R=350$ ), the calculated values of $\% E_{\mathrm{E}}, \%$ $E_{\mathrm{R}}$, average CRLB and $\% E_{\mathrm{R} 2}$ were $-21.8 \%, 9.0 \%, 12.2 \%$ and $7.0 \%$, respectively, when using the default baseline setting of LCModel.

Within-session reproducibility is illustrated in Figure 6 using a box plot, with each box representing four separate GABA measurements from a single subject. The average coefficient of variation across all 14 subjects was $8.7 \pm 4.9 \%$. Also plotted in Figure 6 are the GABA concentration estimates obtained from the full, 192 average, short-TE spectra (black dots). On average, GABA estimates obtained using the full spectra are $14 \%$ lower than those obtained using the partial spectra. This is consistent with the contour plot of $\% E_{\mathrm{E}}$ in Figure $2 \mathrm{a}$, which predicts increasing negative bias in GABA concentration estimates with increasing SNR.

Figure 7 shows two examples of instances in which the $\% E_{\mathrm{R}}$ and CRLB estimates are not indicative of the true reproducibility. In the first example, we consider the data simulated with the broadest LW $(\mathrm{LW}=12 \mathrm{~Hz})$ and the lowest SNR (SNR=50) when analysed using the default baseline setting in LCModel. An example of one of these 500 simulated spectra is shown in Figure 7a. Under these experimental conditions, both $\% E_{\mathrm{R}}$ and the average CRLB are below $20 \%$ (15.9\% and $19.0 \%$, respectively; Fig. 2), indicating relatively reproducible GABA measurements. However, as shown in Figure $7 c$, there appears to be a poor correlation between the estimated versus actual GABA concentrations at this LW and SNR (dots, $R=0.30$ ). Therefore, in this case, the $\% E_{\mathrm{R}}$ and CRLB metrics seem to overestimate the reproducibility of the GABA measurements. In the second example, we consider the data simulated with $\mathrm{LW}=9 \mathrm{~Hz}$ and $\mathrm{SNR}=500$, again when analysed using the default baseline setting in LCModel. An example of one of these 500 simulated spectra is shown in Figure $7 \mathrm{~b}$. Under these experimental conditions, both $\% E_{\mathrm{R}}$ and the average CRLB are above $20 \%$ (23.5\% and $25.4 \%$, respectively; Fig. 2), indicating unreliable GABA measurements. However, as shown in Figure $7 c$, there is a good correlation between the estimated and actual GABA concentrations at this LW and SNR (squares, $R=0.72$ ). Therefore, in this case, the $\% E_{\mathrm{R}}$ and CRLB metrics tend to underestimate the reproducibility of the GABA measurements. 

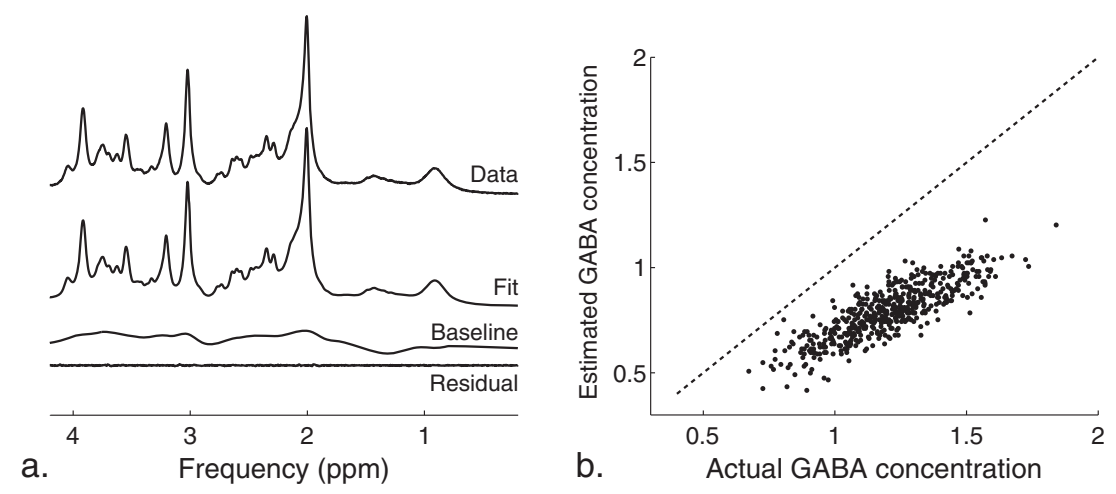

Figure 5. (a) Example simulated data with linewidth (LW) and signal-to-noise ratio (SNR) corresponding to typical experimental conditions observed with the full spin-echo full-intensity acquired localised (SPECIAL) acquisition in the occipital cortex ( $(\mathrm{W}=6 \mathrm{~Hz}$; SNR $=650)$. (b) Scatter plot showing the estimated versus actual $\gamma$-aminobutyric acid (GABA) concentration for all 500 simulated spectra with these experimental conditions.

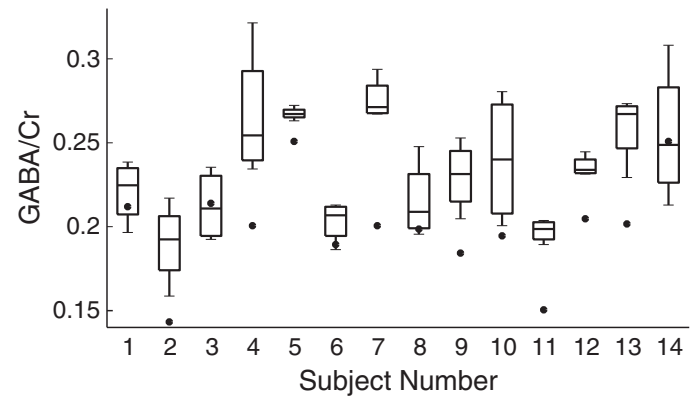

Figure 6. Box plot diagram illustrating within-session reproducibility for all 14 subjects scanned. Box plot data were generated by breaking the short-TE spin-echo full-intensity acquired localised (SPECIAL) acquisition into four separate and consecutive blocks, and analysing each separately. Also shown are the $\gamma$-aminobutyric acid (GABA) estimates from the full SPECIAL acquisition (dots). Cr, creatine.

\section{DISCUSSION}

\section{LCModel baseline}

As discussed in the Methods section, the most appropriate choice of LCModel baseline for the analysis of the simulated data in this study is the option in which the baseline component is omitted from the fit. The contour plots shown in Figure 1 demonstrate that, when the appropriate baseline setting is used, LCModel is able to estimate the GABA concentration with fairly good reproducibility. As the experimental conditions improve (as SNR increases and LW decreases), both the mean estimation error $\left(\% E_{\mathrm{E}}\right.$, Fig. $\left.1 \mathrm{a}\right)$ and the reproducibility errors $\left(\% E_{\mathrm{R}}\right.$, Fig. $1 \mathrm{~b}$; $\% E_{\mathrm{R} 2}$, Fig. 1d) decrease, as expected. The default baseline option in LCModel, however, includes a substantial baseline component in the fit, and is an inappropriate choice for analysis of the simulated spectra in this study, as all of the peaks in the simulated spectra (with the exception of the tail of the residual water peak) should be accounted for by the basis set. Therefore, the contour plots in Figure 2 illustrate the ability of LCModel to reproducibly estimate GABA concentrations, even when an incorrect baseline model is used. The most striking side effect of using an incorrect baseline model is that the mean estimation error no longer decreases as SNR increases (Fig. 2a). Instead, as SNR increases above 200, LCModel begins to underestimate the GABA concentrations, suggesting that, as SNR increases, more and more of the
GABA signal may be incorrectly attributed to the baseline. In addition, the known MM resonances were modelled individually and no constraints were placed on their relative intensities, which may also have contributed to bias in the fit. Measuring the MM baseline explicitly, or constraining the relative intensities of the various baseline components in the model, may help to reduce measurement bias in the fit, and will be the subject of future work. Although the use of an incorrect baseline model appears to have a detrimental effect on the GABA measurement bias, the reproducibility error is not affected as severely. Indeed, provided that SNR is greater than or equal to 150, and LW is less than or equal to $9 \mathrm{~Hz}$ (criteria that can be satisfied by many experimental MRS data), the GABA reproducibility error $\left(\% E_{\mathrm{R} 2}\right)$ remains below $20 \%$, which suggests very good reproducibility despite the use of an incorrect baseline model. To summarise this finding with regard to the LCModel baseline, if the baseline model is not an accurate representation of the actual spectral baseline, LCModel GABA estimates may contain systematic bias, but, provided that the data satisfy basic quality criteria (LW $\leq$ $9 \mathrm{~Hz}$; SNR $\geq 150$ ), simulations indicate that LCModel can provide reproducible estimates of GABA concentration.

\section{Misleading $\% E_{\mathrm{R}}$ and CRLB estimates}

One unexpected finding of this study is that the $\% E_{\mathrm{R}}$ and CRLB estimates are not always indicative of the true reproducibility of the measurement, as shown in Figure 7. To explain these misleading reproducibility error and CRLB estimates, we need to consider that both of these metrics estimate the uncertainty of the measurement relative to the value of the measurement itself. In the case of Figure $7 \mathrm{a}(\mathrm{LW}=12 \mathrm{~Hz}$; $S N R=50)$, the GABA concentrations are greatly overestimated, as can be seen from both the scatter plot in Figure 7c, where the estimated GABA concentrations are much greater than the actual concentrations, and the contour plot of $\% E_{\mathrm{E}}$ in Figure $2 \mathrm{a}\left(\% E_{\mathrm{E}}=+76 \%\right)$. As a result, the measurement uncertainties appear as a small percentage of the average estimated value, resulting in small reproducibility error and CRLB estimates. Conversely, in the case of Figure $7 \mathrm{~b}(\mathrm{LW}=9 \mathrm{~Hz}$; $\mathrm{SNR}=500)$, the GABA concentrations are greatly underestimated $\left(\% E_{\mathrm{E}}=-58.4 \%\right)$, resulting in inflated reproducibility error and CRLB estimates. Therefore, both CRLB and $\% E_{R}$ estimates are influenced by measurement bias, with positive measurement biases tending to result in an underestimation of the CRLB and $\% E_{\mathrm{R}}$ values, and negative measurement biases tending to result 


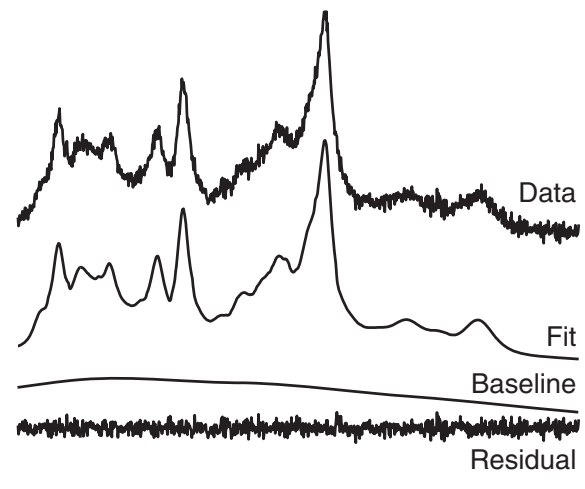

a.
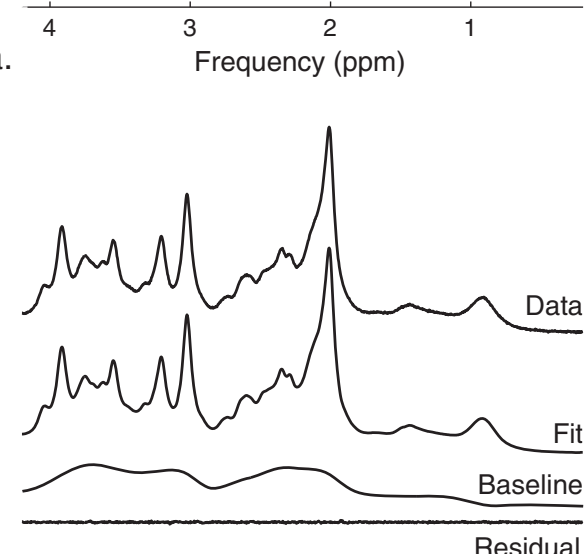

b.
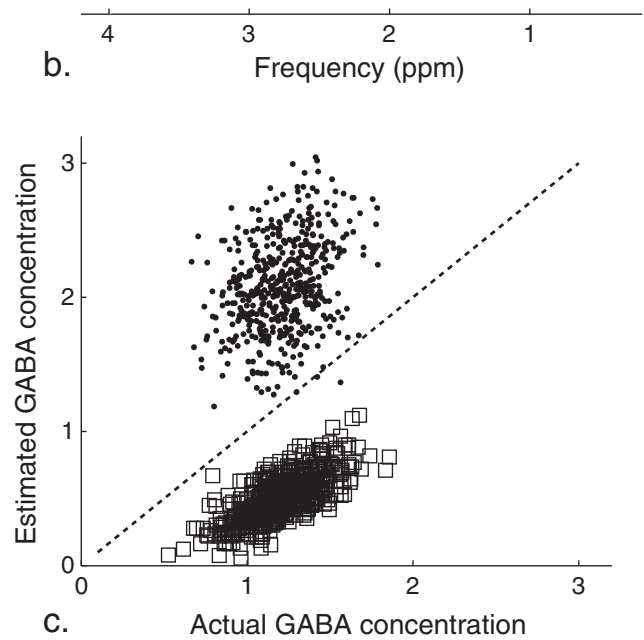

Figure 7. Illustration of misleading Cramer-Rao lower bounds (CRLBs). (a) Example simulated data with linewidth (LW) and signal-to-noise ratio (SNR) corresponding to the worst simulated conditions $(L W=12 \mathrm{~Hz} ; \mathrm{SNR}=50$ ). The average CRLB for simulated data with these experimental conditions was less than $20 \%$, indicating good reproducibility. (b) Example simulated data with $L W=9 \mathrm{~Hz}$ and $S N R=500$. The average CRLB for simulated data with these experimental conditions was greater than $20 \%$, indicating poor reproducibility. (c) Scatter plot showing estimated versus actual $\gamma$ aminobutyric acid (GABA) concentration for all 500 simulated spectra with experimental conditions of (a) (dots) and (b) (squares). Note that, despite the higher CRLB, case (b) appears to show better data reproducibility.

in an overestimation of the CRLB and $\% E_{\mathrm{R}}$ values. For this reason, we have introduced the absolute measure of reproducibility error $\left(\% E_{\mathrm{R} 2}\right)$, which is independent of measurement bias. This metric tends to more accurately reflect the reproducibility error in cases in which there is a large measurement bias. For example, in the case of Figure $7 \mathrm{a}, \% E_{\mathrm{R} 2}$ is $27.4 \%$ and, in the case of Figure $7 \mathrm{~b}$, $\% E_{\mathrm{R} 2}$ is $9.9 \%$. The misleading nature of CRLB estimates suggests that the use of CRLB values as acceptance criteria for MRS metabolite measurements (i.e. CRLB $<20 \%$ ) may not be valid in all situations.

\section{GABA reproducibility under realistic experimental conditions}

The results shown in Figure 5 demonstrate that, under the experimental conditions corresponding to those observed in the in vivo experiments performed here $(\mathrm{LW}=6 \mathrm{~Hz}$; $\mathrm{SNR}=650)$, simulations predict that, although the GABA concentration will be systematically underestimated by approximately $34 \%$, the reproducibility of the measurements $\left(\% E_{\mathrm{R}}=8.9 \%, \% E_{\mathrm{R} 2}=5.9 \%\right)$ is comparable with previously published reproducibility values for edited measurements of GABA at the same field strength $(14,15)$. This high reproducibility is indicated by the strong positive correlation between estimated GABA concentrations and actual concentrations shown in Figure $5 \mathrm{~b}(R=0.84)$. Providing further evidence of the reproducibility of short-TE GABA measurements under these experimental conditions is the observation of a significant positive correlation $(R=0.58, p<0.05)$ between short-TE GABA measurements and gold-standard edited GABA measurements in the same voxel (Fig. 4).

Under the experimental conditions corresponding to the shortened 2.4-min acquisitions performed here $(\mathrm{LW}=6 \mathrm{~Hz}$; SNR $=$ $350)$, simulations predict that GABA concentrations will be systematically underestimated by $21.8 \%$, and that the reproducibility of the measurements $\left(\% E_{\mathrm{R}}=9.0 \%, \% E_{\mathrm{R} 2}=7.0 \%\right)$ is only marginally worse than when using the full 9.6-min acquisition. Furthermore, from the in vivo study of within-session reproducibility, the average coefficient of variation of short-TE GABA measurements was calculated to be $8.7 \pm 4.9 \%$, which agrees well with simulation, and compares favourably with previously published reproducibility values for edited measurements of GABA at the same field strength $(14,15)$.

The in vivo experiments performed here consisted of a shortTE SPECIAL acquisition with a duration of $9.6 \mathrm{~min}$ in a voxel measuring $15.625 \mathrm{~cm}^{3}$. This is fairly representative of a typical short-TE MRS experiment at $3 \mathrm{~T}$, with the exception of the voxel size, which is approximately double the conventional size of $8 \mathrm{~cm}^{3}$ (21). This larger voxel size was chosen to enable matching of the voxel sizes between the short-TE MRS acquisition and the edited experiment, as edited MRS experiments are typically acquired with larger voxels $\left(22.5 \mathrm{~cm}^{3}\right.$ or greater) $(8,12,14)$ because of the relatively low sensitivity of the technique.

It should be noted that the SNR observed when using the shortened 2.4-min acquisition combined with a relatively large $\left(15.625-\mathrm{cm}^{3}\right)$ voxel would be almost identical to the SNR that would be achievable using the more conventional $\sim 10-\mathrm{min}$ acquisition combined with a small $\left(8-\mathrm{cm}^{3}\right)$ voxel. Therefore, one could expect similar reproducibility of GABA estimates under these circumstances. According to the results of both the simulations and the experiments performed here, improvement of the SNR when using the default baseline setting in LCModel tends to result in larger (more negative) measurement bias, with only marginal improvements in reproducibility.

It is recognised that the occipital cortex is one of the more favourable areas of the brain for single-voxel MRS in terms of the LW and SNR that can be typically achieved. Indeed, the SNR achieved in the occipital cortex in this study is higher than 
typical acquisitions in many other brain regions, and it should be emphasised that GABA detection using short-TE MRS may not be reliable in areas of the brain associated with poor shimming and low SNR. Future experimental studies are required to assess the performance of this technique in different regions of the brain.

Because large measurement biases are observed under certain experimental conditions, short-TE MRS at 3T, combined with LCModel analysis, is currently not likely to be a reliable method of obtaining absolute GABA concentration measurements. However, in the vast majority of clinical MRS studies, absolute metabolite concentration measurements are not required. Rather, the main requirement for most studies is the ability to detect changes in metabolite concentrations, or differences between groups. Therefore, measures of reproducibility $\left(\% E_{\mathrm{R}}, \% E_{\mathrm{R} 2}\right)$ are usually the most important quantity for determining the measurement efficacy, and, under the experimental conditions investigated in this study, the values of both $\% E_{\mathrm{R}}$ and $\% E_{\mathrm{R} 2}$ are acceptably small $(<20 \%)$, indicating reproducible GABA measurements.

It should also be noted that, because the measurement bias varies as a function of both LW and SNR, differences in LW and SNR between individual datasets should be viewed as a possible source of error within a study. Therefore, it is very important to ensure similar spectral quality across datasets in a study. This can be achieved by enforcing strict requirements on both the LW and SNR of each dataset, and by rejecting datasets that do not meet these requirements. Furthermore, in studies designed to evaluate correlations between GABA concentrations and other clinical variables, it should be determined whether individual differences in LW and SNR are correlated with the clinical variables. If LW and SNR are correlated with the clinical variables of interest, these parameters should be included as covariates in the statistical analyses.

The simulated spectra in this study were designed to approximate the normal range of metabolite concentrations in a group of healthy humans. In the case of pathology, when one or more metabolites may be outside of the normal range, the results of this study may not be applicable.

The simulated data in this study have been generated using a number of simplifying assumptions. First, as the basis spectra used to fit the simulated data in LCModel were identical to the basis spectra used to generate the simulated data itself, the estimates of measurement bias and reproducibility represent a 'best-case scenario'. In in vivo data, some differences may exist between the actual metabolite signals and the simulated basis spectra used to analyse the data, resulting in some additional quantification errors. However, these differences are assumed to be very small, and this assumption is supported by the excellent correspondence observed between simulated and acquired metabolite signals $(12,25)$. Second, simulated spectra do not take into account certain experimental factors, such as subject motion (and other ghost signals), frequency drift and phase drift. It is assumed that the effect of neglecting these factors is small, and this assumption is supported by the fact that all in vivo acquisitions employed strict pre-processing routines, such as the rejection of motion-corrupted scans and frequency and phase drift corrections to minimise the effects of these factors. Finally, possible differences in $T_{2}$ relaxation rates between individual metabolites were not taken into account in either the generation of the simulated MR spectra or the analysis of simulated and experimental data.

\section{CONCLUSIONS}

Both simulations and in vivo experiments indicate that short-TE SPECIAL MRS is capable of providing reproducible GABA measurements in the occipital lobe at $3 \mathrm{~T}$, even when an incorrect baseline model is used in the analysis of the data. However, the results of the simulations also suggest that the accuracy of GABA concentration estimates obtained with short-TE MRS is dependent on the experimental conditions, and should therefore be used with caution. Future studies are required to determine the efficacy of short-TE MRS for the detection of GABA in other brain regions, especially those associated with poor shim and lower SNR.

\section{Acknowledgement}

This work was funded by the Medical Research Council (G0701421).

\section{REFERENCES}

1. McCormick DA. GABA as an inhibitory neurotransmitter in human cerebral cortex. J. Neurophysiol. 1989; 62(5): 1018-1027.

2. Simister RJ, McLean MA, Barker GJ, Duncan JS. Proton MRS reveals frontal lobe metabolite abnormalities in idiopathic generalized epilepsy. Neurology, 2003; 61(7): 897-902.

3. Sanacora G, Mason GF, Rothman DL, Behar KL, Hyder F, Petroff OA, Berman RM, Charney DS, Krystal JH. Reduced cortical gammaaminobutyric acid levels in depressed patients determined by proton magnetic resonance spectroscopy. Arch. Gen. Psychiatry, 1999; 56(11): 1043-1047.

4. Sanacora G, Gueorguieva R, Epperson CN, Wu YT, Appel M, Rothman DL, Krystal JH, Mason GF. Subtype-specific alterations of gammaaminobutyric acid and glutamate in patients with major depression. Arch. Gen. Psychiatry, 2004; 61(7): 705-713.

5. Donahue MJ, Near J, Blicher JU, Jezzard P. Baseline GABA concentration and fMRI response. Neuroimage, 2010; 53(2): 392-398.

6. Muthukumaraswamy SD, Edden RA, Jones DK, Swettenham JB, Singh KD. Resting GABA concentration predicts peak gamma frequency and $\mathrm{fMRI}$ amplitude in response to visual stimulation in humans. Proc. Natl. Acad. Sci. USA, 2009; 106(20): 8356-8361.

7. Stagg CJ, Bachtiar V, Johansen-Berg $H$. The role of GABA in human motor learning. Curr. Biol., 2011; 21(6): 480-484.

8. Edden RA, Muthukumaraswamy SD, Freeman TC, Singh KD. Orientation discrimination performance is predicted by GABA concentration and gamma oscillation frequency in human primary visual cortex. J. Neurosci. 2009; 29(50): 15 721-15 726.

9. Rothman DL, Petroff OA, Behar KL, Mattson RH. Localized ${ }^{1} \mathrm{H}$ NMR measurements of gamma-aminobutyric acid in human brain in vivo. Proc. Natl. Acad. Sci. USA, 1993; 90(12): 5662-5666.

10. Mescher M, Merkle H, Kirsch J, Garwood M, Gruetter R. Simultaneous in vivo spectral editing and water suppression. NMR Biomed. 1998; 11(6): 266-272.

11. Choi IY, Lee SP, Merkle H, Shen J. Single-shot two-echo technique for simultaneous measurement of GABA and creatine in the human brain in vivo. Magn. Reson. Med. 2004; 51(6): 1115-1121.

12. Near J, Simpson R, Cowen P, Jezzard P. Efficient gamma-aminobutyric acid editing at $3 \mathrm{~T}$ without macromolecule contamination: MEGASPECIAL. NMR Biomed. 2011; 24(10): 1277-1285.

13. Bottomley PA. Spatial localization in NMR spectroscopy in vivo. Ann. NY Acad. Sci. 1987; 508: 333-348.

14. Bogner W, Gruber S, Doelken M, Stadlbauer A, Ganslandt O, Boettcher U, Trattnig S, Doerfler A, Stefan H, Hammen T. In vivo quantification of intracerebral GABA by single-voxel (1)H-MRS-how reproducible are the results? Eur. J. Radiol., 2011; 73(3): 526-531.

15. O'Gorman RL, Edden RA, Michels L, Murdoch JB, Martin E. Precision and repeatability of in vivo GABA and glutamate quantification. Proceedings of the 19th Annual Meeting ISMRM, Montreal, QC, Canada, 2011; 3440.

16. Terpstra M, Ugurbil K, Gruetter R. Direct in vivo measurement of human cerebral GABA concentration using MEGA-editing at 7 Tesla. Magn. Reson. Med. 2002; 47(5): 1009-1012. 
17. Mlynarik V, Cudalbu C, Xin L, Gruetter R. 'H NMR spectroscopy of rat brain in vivo at 14.1 Tesla: improvements in quantification of the neurochemical profile. J. Magn. Reson. 2008; 194(2): 163-168.

18. Tkac I, Andersen P, Adriany G, Merkle H, Ugurbil K, Gruetter R. In vivo ${ }^{1} \mathrm{H}$ NMR spectroscopy of the human brain at $7 \mathrm{~T}$. Magn. Reson. Med. 2001; 46(3): 451-456.

19. Tkac I, Starcuk Z, Choi IY, Gruetter R. In vivo ${ }^{1} \mathrm{H}$ NMR spectroscopy of rat brain at $1 \mathrm{~ms}$ echo time. Magn. Reson. Med. 1999; 41(4): 649-656.

20. Mlynarik V, Gambarota G, Frenkel H, Gruetter R. Localized shortecho-time proton MR spectroscopy with full signal-intensity acquisition. Magn. Reson. Med. 2006; 56(5): 965-970.

21. Mekle R, Mlynarik V, Gambarota G, Hergt M, Krueger G, Gruetter R. MR spectroscopy of the human brain with enhanced signal intensity at ultrashort echo times on a clinical platform at 3T and 7T. Magn. Reson. Med. 2009; 61(6): 1279-1285.
22. Hancu I. Which pulse sequence is optimal for myo-inositol detection at 3 T? NMR Biomed. 2009; 22(4): 426-435.

23. Hancu I, Port J. The case of the missing glutamine. NMR Biomed., 2011; 24(5): 529-535.

24. Govindaraju V, Young K, Maudsley AA. Proton NMR chemical shifts and coupling constants for brain metabolites. NMR Biomed. 2000; 13(3): 129-153.

25. Kaiser LG, Young K, Meyerhoff DJ, Mueller SG, Matson GB. A detailed analysis of localized J-difference GABA editing: theoretical and experimental study at 4 T. NMR Biomed. 2008; 21 (1): 22-32.

26. Naressi A, Couturier C, Castang I, de Beer R, Graveron-Demilly D. Java-based graphical user interface for MRUI, a software package for quantitation of in vivo/medical magnetic resonance spectroscopy signals. Comput. Biol. Med. 2001; 31(4): 269-286. 